\title{
Ausbildungskampagne
}

\section{Pflege braucht Superkräfte}

\begin{abstract}
— Unter dem Motto "Pflege braucht Superkräfte“ hat die St. Vincenz-Krankenhaus GmbH Paderborn eine neue AusbildungsKampagne für den Pflegeberuf gestartet. Headlines wie „Ego kann jeder. Empathie braucht Kraft." oder "Zahlen kann jeder.
\end{abstract}

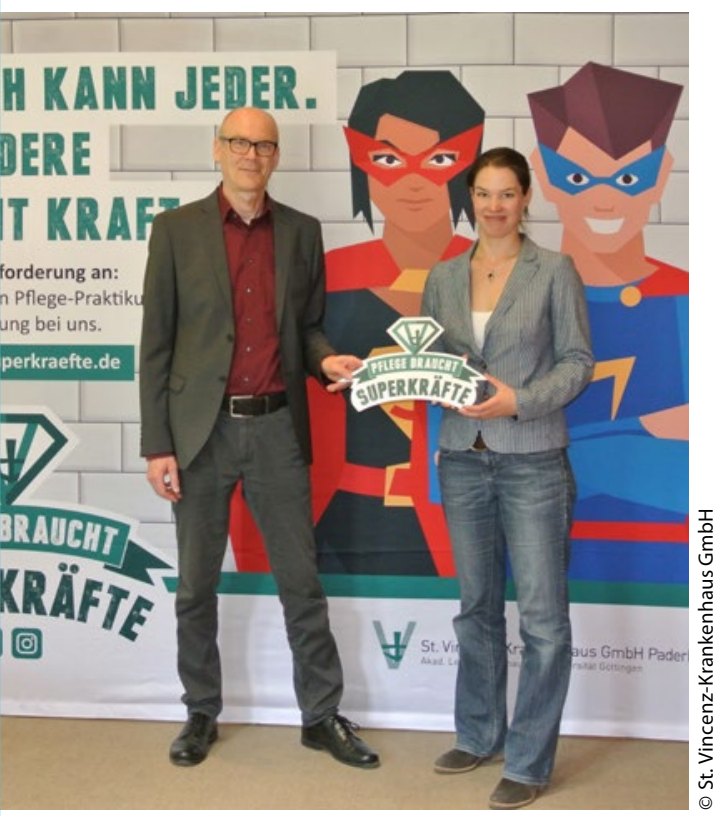

Bildungsreferent Andreas Riekötter und Marketing-Leiterin Annekathrin Kronsbein stellen die neue Kampagne für die Pflegeausbildung vor.
Dankbarkeit gibt Kraft." sowie Superhelden im Comic-Stil sollen junge Menschen für die Pflegeberufe interessieren.

„Kern der Überlegungen war, dass sich Jugendliche im Alltag eher nicht mit Krankheit, Alter und Leid auseinander setzen. Wer sich dem im Krankenhaus freiwillig stellt, verdient also größten Respekt", so Annekathrin Kronsbein, Leiterin Kommunikation und Marketing. Denn dafür seien Mut sowie oft auch körperlich, mental und emotional "übermenschliche" Kräfte erforderlich. Hintergrund ist die Tatsache, dass es immer schwerer wird, junge Menschen für ein Praktikum oder eine Ausbildung in der Pflege zu begeistern. "Schon seit einigen Jahren beobachten wir rückläufige Bewerberzahlen", sagt Bildungsreferent Andreas Riekötter. "Die Pflegeberufe und damit auch die Ausbildung werden in der Gesellschaft ambivalent wahrgenommen: Man hat zwar Vertrauen zu den Pflegenden, verbindet den Beruf jedoch mit schlechter Bezahlung, mangelnden Aufstiegsmöglichkeiten und Überlastung." Die Superhelden der neuen Kampagne sollen dem eine neue Assoziation entgegensetzen: Neben dem "Herz am rechten Fleck" brauche man für die Ausbildung auch Intelligenz und Charakterstärke.

www.pflege-braucht-superkraefte.de
Gemeinsam lernen

\section{Pflegeausbildung interkulturell}

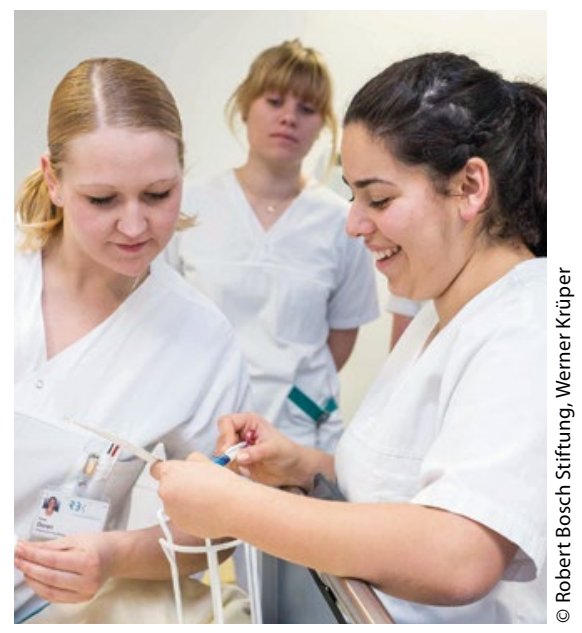

Das Irmgard-Bosch-Bildungszentrum bietet einen besonderen Ausbildungskurs in der Pflege an: Deutsche und Flüchtlinge lernen gemeinsam.

— Im September 2017 startet eine neue, bundesweit einmalige Pflegeausbildung am Irmgard-Bosch-Bildungszentrum des Stuttgarter Robert-Bosch-Krankenhauses. In einer bunt gemischten Ausbildungsklasse lernen Deutsche und Flüchtlinge gemeinsam mehr als theoretisches und praktisches Wissen der Pflege. Ein Viertel der Ausbildung wird sich interkulturellen Themen widmen.

Das Angebot richtet sich an Menschen, die eine Ausbildung in der Gesundheits- und Krankenpflege mit einer interkulturellen Perspektive verknüpfen möchten. Zudem sind Menschen angesprochen, die aus nicht sicheren Herkunftsländern wie Syrien, Iran Irak und Afghanistan fliehen mussten und eine pflegerische Berufsausbildung in Deutschland anstreben. „Eine hohe Bereitschaft zum wechselseitigen Lernen und zur Mitwirkung an einer modellhaften Berufsausbildung sind Voraussetzung“, erklärt Dr. Annette Lauber, Direktorin des IrmgardBosch-Bildungszentrums. „Wir freuen uns, dass bereits viele Bewerbungen von geflüchteten jungen Frauen und Männern unterschiedlichster Herkunftsländer eingegangen sind." Diese sollen nun durch Bewerber aus Deutschland ergänzt werden.

www.rbk.de 